\title{
The Growth of Healthy and Cancerous Tissues
}

\author{
Gyula Peter Szigeti1, Attila Marcell Szasz'2 , Andras Szasz ${ }^{3}$ \\ ${ }^{1}$ Innovation Center, Semmelweis University, Budapest, Hungary \\ ${ }^{2}$ Division of Oncology, Department of Internal Medicine and Oncology, Semmelweis University, Budapest, Hungary \\ ${ }^{3}$ Department of Biotechnics, St. Istvan University, Budaors, Hungary \\ Email: biotech@gek.szie.hu, szasz.andras@gek.szie.hu
}

How to cite this paper: Szigeti, G.P., Szasz, A.M. and Szasz, A. (2020) The Growth of Healthy and Cancerous Tissues. Open Journal of Biophysics, 10, 113-128.

https://doi.org/10.4236/ojbiphy.2020.103010

Received: May 13, 2020

Accepted: June 6, 2020

Published: June 9, 2020

Copyright $\odot 2020$ by author(s) and Scientific Research Publishing Inc. This work is licensed under the Creative Commons Attribution International License (CC BY 4.0).

http://creativecommons.org/licenses/by/4.0/ (c) (i) Open Access

\begin{abstract}
The structure of the tissues is formed in a self-similar manner, forming fractal structures in their transport networks. The structure exhibits allometric forming and so-called scaling behavior. This is a basic growth model fine-tuned by various connections of the cells (junctions and adherent connections), intended to direct material and energy transports between them. This secondary control of cell metabolism decreases primary metabolic transport through the free surfaces of the cells. The cellular network is formed by triggering the endogenous electric fields, which are dominantly governed by cell membrane potential. Proliferation exhibits a different electric pattern due to the low cell-membrane potential and resulting negativity relative to its environment. This potential change characterizes cells in normal proliferation and a cluster of cells (a tumor) in the case of cancerous development. This latter has certain similarities to the leakage transport of liquid in porous media, substituting the pressure with endogenous tumor potential. The average survival of a tumor depends on the kind of available metabolic transport and the fractal dimensions of the newly built angiogenic network.
\end{abstract}

\section{Keywords}

Endogenous Potential, Metabolic Scaling, Competition, Cooperation, Self-Time, Tumor Survival

\section{Introduction}

The form of various organs is evolving in the early stage of the development of the complete body. The evolution of mass of the organ roughly keeps the form-similarity until the adultery of the subject. The question is automatically arising: how the organ grows? We convinced having only one pivotal orientation appears, the bioscaling [1], and the connected ontogenic growth [2], if it is valid 
for the individual organs [3]. In this assumption, we have two consequences:

- The network of the blood-flow in the organ follows the complete network of the body in its structure and organizing,

- The network in the organ is optimized, suitable for the largest available transfer of materials and energy flow.

When dividing the organ on small, tightly connected, space-filling elementary cubes, we may assume that every cube grows isotropic way. The same number of cells is born and dies by apoptosis in average in every cube. Consequently, the linear size of the organ changes by the $1 / 3$ power of time. This model is the equally distributed production of cell numbers. All the cube-elements have the same mass-balance, which has production and annihilation elements (mainly apoptosis) are equal in average in every cube.

There are originally two categories of cells existing by their behaviour: collective and non-collective. The cells in solid malignant tumor usually are non-collective. They are normally autonomic and competing for each other for the necessary nutrients. In the healthy development of the tissue, the cells are cooperative, and this cooperation determines their structural properties. We may assume in healthy cases that every "elementary cube" described above has the same metabolic conditions, [2]; the material transfer for the metabolism, in general, is optimized.

The circulation network is organized by fractal geometry [4], which is based on the unified self-similar construction of the nets. This is a space-filling isotropic growth model with simple steps of building: continuing with the same template at every terminal. Due to this construction procedure, the metabolic rate depends on the 3/4 power of the mass of the given volume [5].

Our goal is to show how the malignant growth modifies the normal healthy growth of the tissue.

\section{Character of the Cell-Fission}

The organ is a set of cells that "democratically" consumes the optimal amount of materials and energy from the network. However, this situation is approximate only. The fractal-network of the vessels, which ensures the distribution is not enough alone to provide the satisfactory info and material-exchange. Therefore, cells form a secondary network of junctions and adherent connections between them, making an additional distribution. These cellular connections make such corrective actions, which guarantee the optimal distribution of the "resources" between the cells. The secondary network needs a part of the membrane surface, decreases the original effective free surface of the cells, decreasing the transport facility forms of the primary network. Accordingly, the self-similar fractal network is not enough for the information exchange and the system forces to generate the secondary networks of the cellular connections. The possible exchange of molecules through the junctions specifies the correction of metabolic processes which is the extension of the primary transport-networks. As well as this secondary (adhesion) structure guarantees the mechanical structure of the 
organ, even it keeps the cells together in its ex-vivo states too.

The logical assumption is that the growth of the consequent effective surface of the cells in a specific organ is proportional to the effective surface of capillaries (a) available for the material transfer. When $N_{c}$ is the actual number of cells, and $a_{c}$ is the effective surface of a cell for metabolic activity, which depends on its environmental population (depends of $N_{c}$ ), so $a_{c}=a_{c}\left(N_{c}\right)$, and $N_{c} \cdot a_{c}\left(N_{c}\right)$ is the effective surface of the input of a single cell, which is of course is limited by the availability of the complete supply (a), so:

$$
a \leq N_{c} \cdot a_{c}\left(N_{c}\right)
$$

We have shown elsewhere [6] that the metabolic rate depends on not only the mass but on the linear size of the circulation length by $3 / 4$ power, too, and the actual average mass $(m)$ and the actual average length of the blood circulatory network, (I), related as:

$$
m \propto K l^{4}
$$

where $K$ is a constant, and $N_{c} \propto m . m \propto L^{3}$, where $L$ is the linear size of the given organ. Using (1) and (2) in case of optimal equality we obtain:

$$
l^{3} \propto l^{4} a_{c}\left(N_{c}\right) \rightarrow a_{c} \propto l^{-1} \propto L^{-3 / 4}
$$

Consequently, the effective surface of a cell cannot decrease more intensive than the $-3 / 4$ power of the linear size of the organ. Hence the effective membrane surface is inversely proportional to the length of the organ, so the size of the growth of the organ allows more possibilities to build up a secondary network between the cells. This has further consequences: when the cell would be able to terminate its secondary network, it will do it because the growth of its active surface allows more metabolic influx. The survival on the cellular level has higher priority for the cell than its organism level.

When the cell division starts, grows its metabolic demand. It needs more energy and material transport than was before, and so the cell increases its demand of metabolic transfer directly through its membrane. It must isolate itself from the network, it tries to reach the autonomy, terminates the surface-limiting connections to neighboring cells. The autonomy of the cells requests a new decision of the fate of it when the division is over, and the two autonomic daughter cells appear. The termination of the networking in this line is an energetic constraint. This well explains the forming of $\alpha$-state [7]. In the case of cancer-state of the cell, the terminated collectivity is one of the factors to fulfill the high metabolic request of permanent growth.

Building up a structure needs stability. The stable state in the level of forces means a stationer equilibrium of the attractive and repulsive forces. Do these forces act in the case of tissues? Yes, these exist in the structure of the tissue. The attractive force is the collectivity. The distance of cellular communication defined by different signaling mechanisms [8], including the intracellular, the information exchange between neighboring cells, as well as short distance communication (e.g. synapses), and long-distance communications by various hor- 
mones and molecules, including exosomes and other vehicles. Research on signaling (social signals) of cells [9] and the pathways of cell-communication are in the focus of emerging attention [10].

There is an attraction between the identical collective cells even from the distance of $\approx 1-4 \mu \mathrm{m}$, [11]. Such long-range interaction could be explained by resonances and super-polarization [12]. The repulsive force which balances the attraction is the membrane polarization; the outside membrane-wall is positive, which repulses the individual cells. The repulsion is crucial in living structures because the material and energy-transport need free surfaces between the cells. The process of decrease in the membrane potential is parallel with the decline in the collective status of the cell.

The formation of the structures has a variation of the symmetries frequently showing 6-fold and 5-fold arrangements. This symmetry-forming is well known in the non-living environment too, [13]. However, the formation of organs is thermodynamically very different from the crystallization of the non-living objects. In the crystallization process heat is liberated permanently because of the crystal-structure forms by the ordering of the disordered material. Consequently, entropy is produced, which must be liberated from the system. The entropy leaves the system by the internal energy flow, heat appears, when the crystallization happens. This mechanism needs a free surface, so the entropy current is limited by the availability of the surface. The information change in living systems is much more than at the crystallization in non-living. A particular temperature does not fix in the living organization, it happens at a wide range of temperature interval, and no permanent cooling is necessary for this organizing process; with another way of entropy decreasing. The entropy-decrease is probably sunk by the order-disorder transition of the aqueous solution; the structured water takes the entropy by its disordering. Without this order-disorder transition of the water, no ordering of the cells could happen. During the organizing process of the cells its structured water transforms to disordered, the water concentration decreases in the cytoplasm, so its resting potential will be higher. Only the cells with resting potential and with ordered water in their interior can be organized. The problem of the cancerous cells is that practically they have no ordered water, which could take the entropy of the ordering transition of the cell. When this primary ordering is not able to be performed, the polymerization forming of the cytoskeleton is also blocked.

The energy minima derive the actual arrangement of the non-living structures. Contrary, the living ones prefer the symmetries depending on the real role of the collectivity of the cells. The structures which must have small hydraulic resistance for transfers will arrange mostly pentagonal (five-fold) symmetry which has "lazy" space filling, allow free space for transfers [14]. However, when the surface energy should be minimized, the 6-fold, hexagonal structure forms.

The above forming symmetry well explained by the Dirichlet theorem of minimizing the electrostatic energy, [15]. Consider the cells conductive, having resting potential $(70-100 \mathrm{mV})$. There is neutral (having the same amount of 
positive and negative ions) electrolyte between the cells. However, the equilibrium ion-distribution of the extracellular electrolyte changes nearby the cells, electric double-layer forms, Figure 1. The substitutional circuit on discrete capacitors was worked out in details [16].

The tissue contains a network of capacitors, as many parallel circuits as the number of cells in Figure 2.

According to the Dirichlet theorem, the electrostatic energy has its minimum in the equilibrium phase. The energy could be minimized, when the complete, consequent capacity of the system in Figure 2 is minimal. The capacity depends on the permittivity of the material between the electrodes and the geometry of the electrode arrangement. Knowing that the thickness of the double layer and inter-distance of the cells are much less than the size of the cell, the condensers could be regarded as planar, so

$$
\frac{A}{d}
$$

where $A$ is the value of the surface, and $d$ is the distances of the cells (electrodes of the capacitors). The coagulation of the cells is inhibited by their surface charges. Consequently, the minimizing of the consequent capacity could happen by the minimizing of the actual surfaces or maximize the distances. In the case of a hexagonal arrangement, the surface would be kept to a minimum; however, with a pentagonal structure thickness increases. Which solution is realized depends on the actual function of the arrangement.

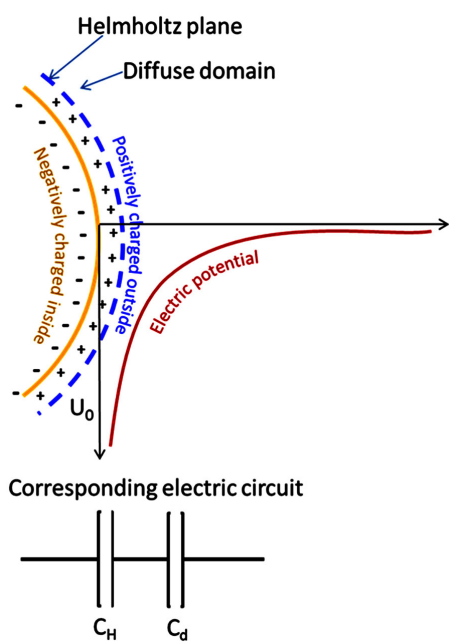

Figure 1. Distribution of charge and potential near the boundary of the cell.

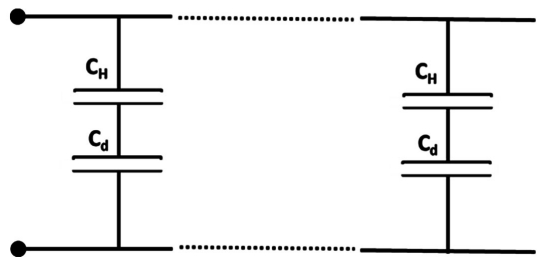

Figure 2. Electric substitutional picture of the tissue. 
The physics of the tissue forming in this picture is very like the stability conditions of the colloidal solutions. This description well fits to the early evolution, when the coagulation is prohibited, and the free-space between the cells was governed by the metabolic transports. The formed distances depended on the shape, resting potential, charge-conditions, etc.

The above description models some tendencies but cannot explain the forming of the tissue by mostly repulsive interactions. The opposite (attractive) forces have to be involved for more realistic description. This request is like the forming of non-living structures, but the non-living explanations could not be adequate to the active living state. One of the guiding rules of the non-living world is the tendency to make energy-minima. For example, the crystals show this rule. The crystals, however, have no unique forms; they could have very different crystalline structures. All the crystalline structures obey the minimal energy rule; however, various tissues form in the living cell based on the nature of that particular organ. The organs have appropriate forms and their size growth by time. The tissue structure of the organs also has an identity; their cells form the tissues of the organs with the unified architecture. By the growing of the subject, the architecture grows too, but the shape is conserved. The form of the organ, tissue, and cells could not be explained by physical interaction mechanisms alone. The form must be functional. The functional architecture probable was formed successively by the evolution, and the mutations, the selection and their information fixed in the DNA developed the self-replication basic mechanisms.

The function of the cell is determined by its position in the tissue, so probably two identical cells do not exist. The cell has adaptability and differentiability making it suitable for the actual function of the actual position. The cell behaves differently when the conditions change. In this meaning, it is well like a logical electric circuit. The basic discrete elements are equivalent, but their function differs according to their actual position in the circuit. Its actual environment determines the differentiation of the cell. The environment by its physical and chemical inputs reprograms the cellular function, fixing it in the DNA. This also means that certain useless functions are blocked. The newly born cell inherits this block. This is the microscopic evolution realizing the function of the cell in the organ according to its position. The macro-differentiation develops the basic cells of the organs.

The growth of the organ keeps the geometric similarity, accompanied by the fixed density in actual place of the organ. Probable the structure does not change inside of the organ while it is healthy, hence the cell-fission is homogeneous ligands, and division promoters distribute homogeneously in, they have gradient only at the boundary of the organ.

\section{Growth of a Tumor and Its Average Survival Time}

Before you begin to format your paper, first write and save the content as a separate text file. Keep your text and graphic files separate until after the text has been formatted and styled. Do not use hard tabs, and limit use of hard returns to 
only one return at the end of a paragraph. Do not add any kind of pagination anywhere in the paper. Do not number text heads-the template will do that for you.

The ratio of $l$, and the asymptotic length of completed network $\left(l_{0}\right)$, equal with the ratio of $m$ and the asymptotic mass $\left(m_{0}\right)$ on the $1 / 4$ power, so

$$
\left(\frac{m}{M}\right)^{1 / 4}=\frac{l}{l_{0}}=\lambda
$$

Consequently, the average $l$ length of the network may be chosen as growth factor. We may introduce the biological self-time by

$$
\begin{aligned}
& \lambda(\tau)=1-\mathrm{e}^{-\tau} \\
& \tau=a t / 4 M^{1 / 4}-\ln \left(1-\lambda_{0}\right)
\end{aligned}
$$

where $\lambda_{0}$ is the ratio of the just-born mass to the asymptotic (completed, final) mass, and $\tau$ is the self-time. This well corresponds with the measurements, [17]. Every similar network has same biological self-time.

The normal reaction to injury is to improve the cellular proliferation replacing the damaged cells and heal the wound. The membrane potential of these newly proliferation cells is also significantly lower than the cell membrane potential of healthy mature cells [18]. After maturing of the daughter cells, their membrane-potential grow again to the healthy character. However, in cancerous proliferation, such return does not happen. The mineral contents of the cancerous cells are robustly changed which has role keeping the cell-membrane potential low, [18] [19] [20], especially an increase in the intracellular concentration of positively charged ions and an increase in negative charges on the glycocalyx coat of the cell. Together with the lower electrical membrane potentials cancer cells have lower electrical impedance than normal cells [20] [21] [22].

The membrane potentials of proliferating cells are certainly lower, irrespective that these are malignant or normal processes [23] [24], as well as the macrophages seeking to repair the broken arrangements changes its membrane potential is not repulsed by the irregularly depolarized, out of network cooperation cells [25] Figure 3.

Membrane potential of cells $\left(V_{m}\right)$

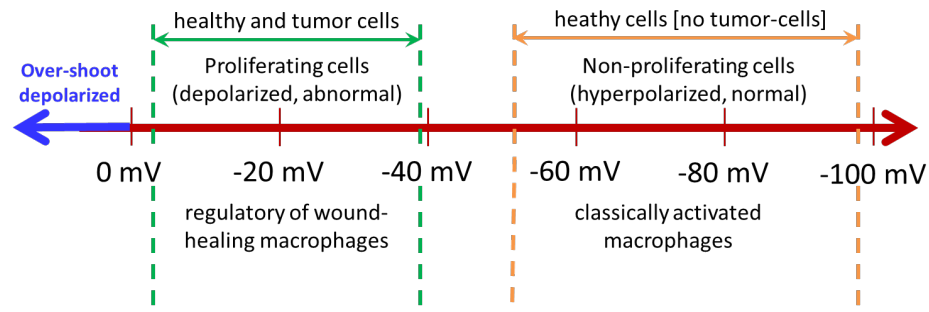

Figure 3. Membrane potentials of networked and proliferating cells are forming two distinct groups. The proliferation (irrespective that it is healthy or malignant) is always depolarized. Eg. the fertilized egg has approximately the same membrane potential $(\mathrm{Vm})$ than the ovarian tumour-cells; mouse hepatoma cells have approx. the same Vm than the human one; proliferating fibroblasts and fibrosarcoma have also approx.. same Vm. 
Therefore, a key component of cell repair and cancer treatment would be to re-establish a healthy membrane potential in the body's cells [26] [27]. The external oscillating electric field in the extracellular electrolyte can adversely affect and support the growth of the membrane potential, [28] [29]. This is one of the factors of modulated electrohyperthermia ( $\mathrm{mEHT}$, trade name oncothermia) [30] [31].

\section{Growth of the Healthy Tissues, Competition for Nutrients}

The mechanisms of the growth of the cellular clusters are very similar for cancerous and healthy embryonal cases. The huge difference, however, in their boundary conditions. A tumor grows dominantly on its surface, while the growth of normal embryo is certainly volumetric. Consequently, the supportive transport network has different fractal structure.

The embryonic growth of healthy organ performs cell division on any points of the organ volume. This growth results in embryonic cells, sharing the same terminal of the blood-vessel network as the mother cell had originally. Due to the relative negative surface (lowered positive charges) of the embryonal cells, a potential gradient will induce currents activation the VEGF receptors, triggering the directed growth of the transport network to serve them [32]. During the transition time, until the generation of the new blood vessels, the diffusion transports the nutrients, governed by the concentration gradients. Note, that the physiological electric fields can generate not only neo-vascular growth but directional nerve development as well [33]. The triggering and control of the construction of various networks in the living systems could be well organized by endogenous electric signals [34]. These processes belong to the category of diffusion-limited aggregation (DLA), like the electric field of dielectric breakdown production too [35]. The DLA processes produce branching pattern fractal network [36].

The process of growing number of cells is not a competition; it is the repetition on the same physiological principle in all steps of the development. The process is stable because the mature cell stops the triggering. The primary embryonal cell starts the forming of its network (electric field triggering, like a template) and this micro-process repeated identically, constructs the self-similar structure, serving the formed network with well-supplied blood.

\section{Growth of Cancer}

The cancerous set of cells has in principle infinite ability of growth. This agglomerate is more negative in its charge than the healthy surrounding because the individual cells are negatives. This macroscopic cluster similarly triggers the growth of blood-supply as the individual embryonic cell, but this is now macroscopic. The growth of this macroscopic set of cells is surface controlled, (the surface of the cluster growths), while the embryonic process is volume controlled by microscopic source. Consequently, despite the applied same physio- 
logical principle the branching pattern and so the fractal dimension will be different. In the case of cancer, the layers are growing on each other. The layer of a surface neo-angiogenic fractal is covered by the next layer of neo-angiogenic fractal supplying the tumor layer by layer.

The Darcy-principle describes the velocity of the liquid in leakage [37]. The mathematical form from Hagen-Poisseuil and Navier-Stokes equations

$$
\bar{v}=\mu \operatorname{grad} p
$$

where $p$ is the pressure. In aqueous solutions like the blood, the incompressibility of the liquid is assumed, so

$$
\operatorname{div} \bar{v}=0
$$

Hence the pressure satisfies the Laplace equation:

$$
\Delta p=0
$$

Which is completed by boundary conditions, which has a definite role in the forming of the branching pattern, but in our present mathematical process have no role.

The triggering electric field acts in porous media too, where the differential Ohm-law is effective:

$$
\bar{j}=\sigma \bar{E}
$$

where $\bar{j}$ is the current density and $\bar{E}$ is the electric field-strength.

Due to the relatively large conduction of the extracellular electrolyte, no space-charge could be formed. Hence

$$
\operatorname{div} \bar{j}=0
$$

On the other hand, the field is induced by standing charges, so it is curl-free:

$$
\bar{E}=\operatorname{grad} \Phi
$$

Accordingly, the formed electric potential satisfies the Laplace equation:

$$
\Delta \Phi=0
$$

Compared this to (9) shows the mathematical equivalency of the electric trigger of this phenomenon with the leakage problem in space.

Consequently, from the above, while the angiogenesis stops by the maturation of the healthy cell, in the case of the malignancy the neo-angiogenesis remains active until the nutrients input balances the requested amount of keeping the tumor alive. When the balance is created, no further growth happens. However, during the permanent division of tumour-cells, the electric trigger remains active, and the balance of the amount of transport and utilization cannot be created.

The nutrition influx made by transport of the vascular-network is proportional a power of the number of the cells which are alimented, due to the self-similarity of the construction process. This power exponent appears as the fractal character of the pattern of the actually formed vascular-network.

The physical mechanism of structural developing of the cells is probably iden- 
tical or very similar to the healthy and tumorous growth. The tree-branch-like structure of the leakage currents is a morphogenetic stimulus. The crucial factor is the fission of the cells. During the division, the cell isolates itself from the standard healthy network. By this isolation, its effective membrane surface growth and the mobility of ionic species (nutrients and waste) grow as well. The cells in fission have definitely smaller membrane potential than the same cell in the healthy network [23]. This relatively negative charge is essential for the start of flows of micro-currents. Furthermore, the individuality constructs a freedom of their shapes (which follows the collapse of the normal cytoskeleton during the fission), and usually, they are more globular than there are in networks. With this, the cells in fission minimize their volumetric energy and maximize their relative surfaces for metabolic transfers. During the fission process, the free extracellular electrolyte in the vicinity of the cell allows higher diffusion rates of ions and molecules and could happen more glucose supply from and more waste efflux to the neighborhood. These processes support the drastic increase in the energy demand of the division, producing two daughters.

In the case of tumor development, the cluster of the dividing cells (micro-tumour) has relative negative potential on its cluster (a tumor) surface due to the relative negativity of the surface of the cells inside the cluster. The surface of starting tumor by developing micro-cluster remains negative, which induces the development of new blood vessels. The cancerous cluster of cells has a united action to create the potential for angiogenesis; the demand for nutrients is collective, while the healthy cells are forming their optimal pattern individually. The neo-angiogenesis dominantly supplies the tumor surface. The development of the new vessels occupies the surface layer of a tumor [38]. Inside of the cluster mostly has less oxygen, and starts the fermentative ATP generation, like in healthy cases by hypoxia.

In many cases, the expected survival time of cancerous patients is approximated from the doubling-time of tumor size [7]. In the following, we use this approximation, and transform the allometric parameter from the mass [2] to the characteristic size in the scaling, using the experimentally easy measurable $L_{0}$ linear size of the tumorous cell-cluster.

Similarly, to (2) the connection of the mass and its characteristic length of the transport network have scaling of fourth power, so

$$
m_{t}=K_{t} l_{t}^{4}
$$

where $K_{t}$ is constant and $l_{t}$ is the characteristic (average) length of the transport network (dominantly neo-angiogenic) and $m_{t}$ is the actual mass of a tumor. Due to the dense surface growth of the new vessels, we can consider its asymptotic structure by Hilbert fractal [39], having fractal dimension [40]. This means the linear size of a tumor $\left(L_{t}\right)$ is proportional with $l_{t}$, so the linear size of the tumour determines the mass by scaling:

$$
m_{t}=K_{0} L_{t}^{4}
$$

where $K_{0}$ is a constant, which value depends on the structure and density of 
the tumour. When the asymptotic mass is $M_{t}$ and the asymptotic linear size of the tumour is $L_{t 0}$, then:

$$
M_{t}=K_{0} L_{t 0}^{4}
$$

moreover, consequently:

$$
\left(\frac{m_{t}}{M_{t}}\right)^{\frac{1}{4}}=\frac{L_{t}}{L_{t 0}}
$$

From (6) we get that the geometric size of growing could be expressed as a universal expression like:

$$
\begin{aligned}
& \frac{L_{t}(\tau)}{L_{t 0}}=1-\mathrm{e}^{-\tau} \\
& \tau=\frac{a t}{4 M_{t}^{\frac{1}{4}}}-\ln \left(1-\frac{L_{t}(\tau=0)}{L_{t 0}}\right)=\frac{a t}{4 K_{t} L_{t 0}}-\ln \left(1-\frac{L_{t}(\tau=0)}{L_{t 0}}\right)
\end{aligned}
$$

When the size of a tumor is $L_{\text {detec }}$ in the actual time of detection, and the lethal size is approximated as $L_{\text {leth }}$ both parameters are experimentally determined.

Assuming that the asymptotic size is longer than both the detected and lethal sizes, then the exponential function well approximated by the two first terms of its Taylor series, like:

$$
\langle t\rangle:=\left(t_{\text {leth }}-t_{\text {detec }}\right)=\frac{4 K_{t}}{a}\left(L_{\text {leth }}-L_{\text {detec }}\right)
$$

This is valid for ideal alimentation of a tumor, so here $\langle t\rangle=\langle t\rangle_{i d}$. The formulation shows definite similarities with the formulation of survival time by approximation with diffusion theory [41].

As we had shown elsewhere, the growth of the mass of a tumor in case of non-ideal alimentation (shortage of nutrients) support is described with the formulation:

$$
\frac{\mathrm{d} m_{t}}{\mathrm{~d} t}=a m_{t}^{\alpha}\left(1-\left(\frac{m_{t}}{M_{t}}\right)^{1-\alpha}\right) \rightarrow \frac{\mathrm{d}\left(\frac{m_{t}}{M_{t}}\right)^{1-\alpha}}{\mathrm{d} t}=\frac{a(1-\alpha)}{M_{t}^{1-\alpha}}\left(1-\left(\frac{m_{t}}{M_{t}}\right)^{1-\alpha}\right)
$$

Hence its solution:

$$
\begin{aligned}
& \left(\frac{m_{t}}{M_{t}}\right)^{1-\alpha}=1-\left(1-\left(\frac{m_{t 0}}{M_{t}}\right)^{1-\alpha}\right) \mathrm{e}^{-\frac{a(1-\alpha) t}{M_{t}^{1-\alpha}}}=1-\mathrm{e}^{-\frac{a(1-\alpha) t}{M_{t}^{1-\alpha}}+\ln \left(1-\left(\frac{m_{t} 0}{M_{t}}\right)^{1-\alpha}\right)}=1-\mathrm{e}^{-\tau}, \\
& \tau=\frac{a(1-\alpha) t}{M_{t}^{1-\alpha}}-\ln \left(1-\left(\frac{m_{t 0}}{M_{t}}\right)^{1-\alpha}\right)
\end{aligned}
$$

where $\alpha$ depends on the fractal dimension of the vascular-network.

Usually, in healthy structures, the fractal dimension of the length of the transport network is 1 , the effective surface in this state has fractal dimension 3 , 
and the volume where it fills up has dimension 4 . In the tumor case, the linear fractal dimension is not valid ever more; the angiogenic structures cover the surface of a tumor densely. In measurements, the fractal dimension of the transport network in cancer is higher than 1. In the asymptotic situation, we expect complete covering with Hilbert fractal pattern with dimension 2 of the network pattern. In the case of DLA in 3-dimension is about 2.5 [42], in plane growth 1.41, [43].

Let us denote by $\varepsilon$ the increase in transport-network dimension from 1 . In this last case,

$$
\alpha=\frac{3-\varepsilon}{4+\varepsilon}
$$

which is in plane DLA growth case $\varepsilon=0.41$, so $\alpha=\frac{2.59}{4.41} \approx 0.587$.

Hence:

$$
\begin{aligned}
& \left(\frac{L_{t}(\tau)}{L_{t 0}}\right)^{1+\varepsilon}=1-\mathrm{e}^{-\tau} \\
& \tau=\frac{a \frac{1+\varepsilon}{4+\varepsilon} t}{M_{t}^{1+\varepsilon}}-\ln \left(1-\left(\frac{L_{t}(\tau=0)}{L_{t 0}}\right)^{1+\varepsilon}\right)=\frac{a \frac{1+\varepsilon}{4+\varepsilon} t}{K_{t} L_{t 0}^{1+\varepsilon}}-\ln \left(1-\left(\frac{L_{t}(\tau=0)}{L_{t 0}}\right)^{1+\varepsilon}\right)
\end{aligned}
$$

When the actually detected linear size of the cancer is again $L_{\text {detec }}$ and $L_{\text {leth }}$ is the lethal size, both have to be determined in every tumour-type on the experimental way. When the asymptotic size is considerably more than both, the average survival time in case of non-ideal alimentation in optimized support of the complete volume $\left(\langle t\rangle_{\text {nidopt }}\right)$ with the available nutrients:

$$
\langle t\rangle_{\text {nidopt }}:=\left(t_{\text {leth }}-t_{\text {detec }}\right)=\frac{K_{t}}{a} \frac{4+\varepsilon}{1+\varepsilon}\left(L_{\text {leth }}^{1+\varepsilon}-L_{\text {detec }}^{1+\varepsilon}\right)
$$

This survival time shows the non-ideal (shortage) supply of alimentation but the available nutrients as maximal as possible [2]. In this case, the boundary conditions govern the process which does not distribute the available nutrients optimally in a complete tumour, prefers the outer layers supplied better by the neo-angiogenic formations. This calculation shows the considerable difference from the approximated average survival time by diffusion theory [41].

Another approach of non-ideal alimentation is when we assume the ideal distribution of the available nutrients in all over the volume. In this case, we have four-dimensionality of the dependence of metabolism from the mass [44]. In this case

$$
\alpha=\frac{3-\varepsilon}{4}
$$

Hence

$$
m=K L_{0}^{1+\varepsilon}
$$

Moreover, consequently: 


$$
\langle t\rangle_{\text {nonid } 4}(\varepsilon):=\left(t_{\text {leth }}-t_{\text {detec }}\right)=\frac{K_{t}}{a} \frac{4}{1+\varepsilon}\left(L_{\text {leth }}^{1+\varepsilon}-L_{\text {detec }}^{1+\varepsilon}\right)
$$

This calculation shows considerable difference again from the approximated average survival time by diffusion theory.

Comparison of survival times in healthy, ideal and cancerous, non-ideal alimentation conditions it is evident that the survival time decreases by the growing limitation of nutrients. Survival time vs. the fractal dimension, which characterizes the degree of non-ideal conditions, shows a rapid decline in survival by growing fractal dimension, Figure 4.

In the case of an optimal distribution of nutrients throughout a tumor, the decrease in survival is slightly more than in the situation of heterogeneity, which maximizes the metabolic rate of a tumor. In the case of simple DLA, forming the survival of maximized and ideal metabolic conditions is $32.5 \%$ and $29.5 \%$, respectively. In the case of the complete surface covering (a blood-pool) when a Hilbert fractal is formed (most advanced cancer cases), the corresponding values are $6.9 \%$ and $5.5 \%$.

\section{Conclusion}

The primary metabolic transport is governed by the free membrane surface of the cell. This free surface is suppressed by various connections of the cells (junctions and adherent connections), intended to additional material and energy transports. This precise control of cell metabolism decreases primary metabolic transport through the free surfaces of the cells. The cellular network is formed by triggering the endogenous electric fields, which are dominantly governed by cell

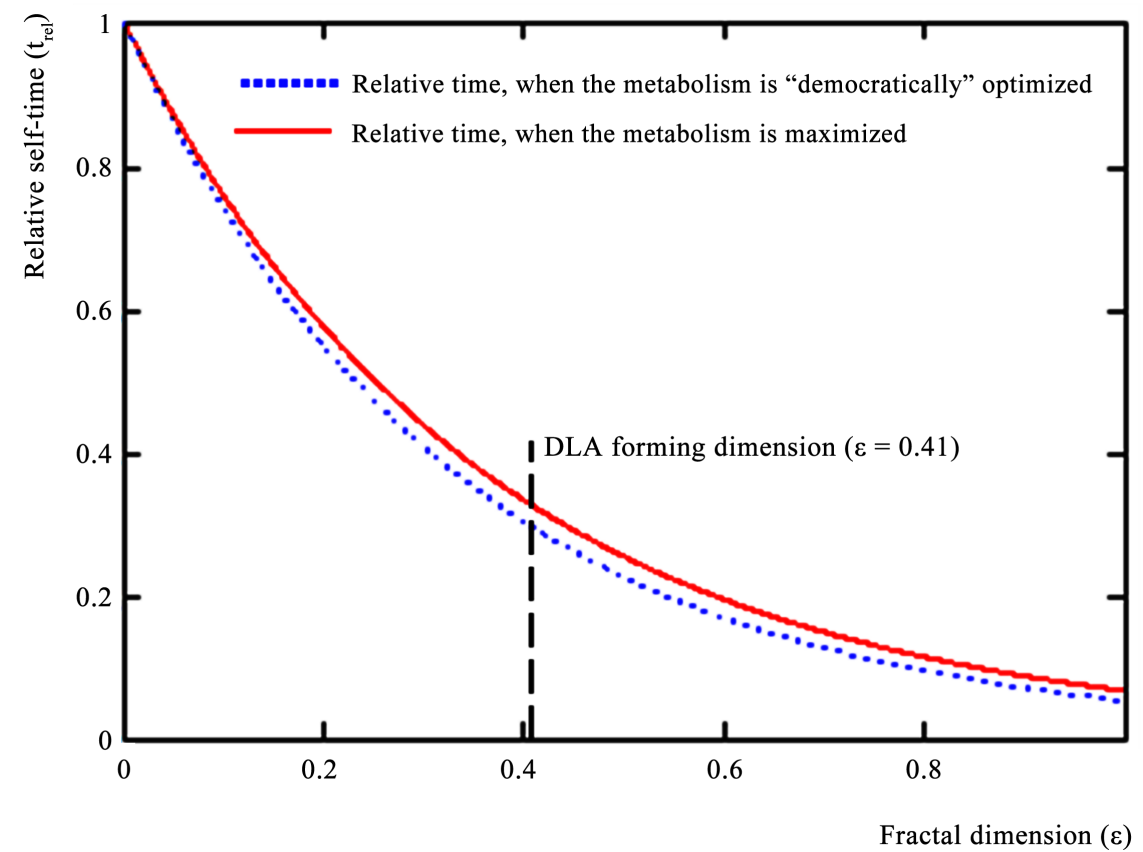

Figure 4. The relative survival time vs. fractal dimension (changing of limiting the alimentation conditions), shows shortening of survival by the development of a tumor. 
actual membrane potential. The proliferation of the cell occurs with low cell-membrane potential resulting negativity relative to its environment. Cancer-cells break their cellular connections "free" their membrane surface for intensive metabolic activity, mainly by fermentative, anaerobic way (Warbug effect [45]), and also these cells have lower membrane potential than their healthy counterparts. The malignant proliferation has certain similarities to the leakage transport of liquid in porous media, substituting the pressure with endogenous tumor potential. The average survival of the tumour-cells shortens by the growing fractal dimension of the newly built angiogenic network and modifies by the kind of alimentation of the tumour.

\section{Acknowledgements}

This work was supported by the Hungarian National Research Development and Innovation Office KFI grant: 2019-1.1.1-PIACI-KFI-2019-00011.

\section{Conflicts of Interest}

The authors declare no conflicts of interest regarding the publication of this paper.

\section{References}

[1] Vicsek, T. (2001) Fluctuations and Scaling in Biology. Oxford University Press, Oxford.

[2] West, G.B., Brown, J.H. and Enquist, B.J. (2001) A General Model for Ontogenic Growth. Nature, 413, 628-631. https://doi.org/10.1038/35098076

[3] Calder, W.A. (1996) Size, Function and Life History. Dover Publ. Inc., Mineola.

[4] Gazit, Y. (1996) Fractal Vasculature and Vascular Network Growth Modeling in Normal and Tumor Tissue. PhD Thesis.

[5] West, G.B., Woodruff, W.H. and Brown, J.H. (2002) Allometric Scaling of Metabolic Rate from Molecules and Mitochondria to Cells and Mammals. PNAS, 99, 2473-2478. https://doi.org/10.1073/pnas.012579799

[6] Szasz, O. and Szigeti, Gy.P. (2020) Allometric Scaling by the Length of the Circulatory Network. Frontiers in Physiology Fractal and Network Physiology. (Under Review)

[7] Szentgyorgyi, A. (1978) The Living State and Cancer. Marcel Dekker Inc., New York.

[8] Brücher, B.L. and Jamall, I.S. (2014) Cell-Cell Communication in the Tumor Microenvironment, Carcinogenesis, and Anticancer Treatment. Cellular Physiology and Biochemistry, 34, 216-243. https://doi.org/10.1159/000362978

[9] Raff, M.C. (1992) Social Controls on Cell Survival and Death. Nature, 356, 397-400. https://doi.org/10.1038/356397a0

[10] Ferrarelli, L.K. (2014) Refining the War on Cancer. Science Signaling, 7, eg2. https://doi.org/10.1126/scisignal.2005276

[11] Nir, S. and Andresen, M. (1977) Van der waals Interactions between Cell Surfaces. The Journal of Membrane Biology, 31, 1-18. https://doi.org/10.1007/BF01869396

[12] Pokorny, J., Hasek, J., Vanis, J. and Jelinek, F. (2008) Biophysical Aspects of Can- 
cer-Electromagnetic Mechanisms. Indian Journal of Experimental Biology, 46, 310-321.

[13] Vincze, Gy., Zsoldos, I. and Szasz, A. (2004) On the Aboav-Weaire Law. Journal of Geometry and Physics, 51, 1-12. https://doi.org/10.1016/j.geomphys.2003.08.003

[14] Szasz, A. (1991) An Electrically Driven Instability: The Living-State (Does the Room temperature Superconductivity Exist?). Physiological Chemistry and Physics and Medical NMR, 23, 43-50.

[15] Grinhspan, A. (2001) A Minimum Energy Problem and Dirichlet Spaces. Proceedings of the American Mathematical Society, 130, 453-460.

[16] Grodzinsky, A.J. (1998) Fields, Forces and Flows in Biological Tissues and Membranes MIT.

[17] Bru, A., Albertos, S., Subiza, J.L., Asenjo, J.L.G. and Bru, I. (2003) The Universal Dynamics of Tumor Growth. Biophysical Journal, 85, 2948-2961. https://doi.org/10.1016/S0006-3495(03)74715-8

[18] Cone, C.D. (1975) The Role of Surface Electrical Transmembrane Potential in Normal and Malignant Mitogenesis. Annals of the New York Academy of Sciences, 238, 420-435. https://doi.org/10.1111/j.1749-6632.1974.tb26808.x

[19] Cone, C.D. (1970) Variation of the Transmembrane Potential Level as a Basic Mechanism of Mitosis Control. Oncology, 24, 438-470. https://doi.org/10.1159/000224545

[20] Cone, C.D. (1985) Transmembrane Potentials and Characteristics of Immune and Tumor Cells. CRC Press, Boca Raton.

[21] Blad, B. and Baldetorp, B. (1996) Impedance Spectra of Tumour Tissue in Comparison with Normal Tissue: A Possible Clinical Application for Electrical Impedance Tomography. Physiological Measurement, 17, A105-A115. https://doi.org/10.1088/0967-3334/17/4A/015

[22] Stern, R.G. (1999) Carcinogenesis and the Plasma Membrane. Medical Hypotheses, 52, 367-372. https://doi.org/10.1054/mehy.1997.0657

[23] Levin, M. (2007) Large-Scale Biophysics: Ion Flows and Regeneration. Trends in Cell Biology, 17, 261-269. https://doi.org/10.1016/j.tcb.2007.04.007

[24] Yang, M. and Brackenbury, W.J. (2013) Membrane Potential and Cancer Progression. Frontiers in Physiology, Membrane Physiology and Membrane Biophysics, 4, Article No. 185. https://doi.org/10.3389/fphys.2013.00185

[25] Erndt-Marino, J. and Hahn, M.S. (2016) Membrane Potential Controls Macrophage Activation. Frontiers in Bioengineering and Biotechnology, 4, pages. https://doi.org/10.3389/conf.FBIOE.2016.01.00360

[26] Alexander, A.D. (1997) Calcium 2-AEP and Calcium Orotate Found Essential in the Prevention and Treatment of Osteoporosis. INI Newsletter June.

[27] Nieper, H.A., Alexander, A.D. and Eagle-Ogden, G.S. (1999) The Curious Man: The Life and Works of Dr. Hans Nieper. Avery Publishing Group, Garden City Park.

[28] Marszalek, P., Liu, D.-S. and Tsong, T.Y. (1990) Schwan Equation and Transmembrane Potential Induced by Alternating Electric Field. Biophysical Journal, 58, 1053-1058. https://doi.org/10.1016/S0006-3495(90)82447-4

[29] Tsong, T.Y. (1990) Electrical Modulation of Membrane Proteins: Enforced Conformational Oscillations and Biological Energy and Signal Transductions. Annual Review of Biophysics and Biophysical Chemistry, 19, 83-106. https://doi.org/10.1146/annurev.bb.19.060190.000503

[30] Fiorentini, G. and Szasz, A. (2006) Hyperthermia Today: Electric Energy, a New 
Opportunity in Cancer Treatment. Journal of Cancer Research and Therapeutics, 2, 41-46. https://doi.org/10.4103/0973-1482.25848

[31] Vincze, Gy. and Szasz, A. (2015) Reorganization of Actin Filaments and Microtubules by outside Electric Field. Journal of Advances in Biology, 8, 1514-1518.

[32] Zhao, M., et al. (2004) Electrical Stimulation Directly Induces Pre-Angiogenesis Responses in Vascular Endothelial Cells by Signaling Trough VEGF Receptors. Journal of Cell Sciences, 117, 397-405. https://doi.org/10.1242/jcs.00868

[33] Song, B., Zhao, M., John, F. and McCaig, C. (2004) Nerve Regeneration and Wound Healing Are Stimulated and Directed by an Endogenous Electrical Field in Vivo. Journal of Cell Science, 117, 4681-4690. https://doi.org/10.1242/jcs.01341

[34] Levin, M. (2014) Molecular Bioelectricity: How Endogenous Voltage Potentials Control Cell Behavior and Instruct Pattern an Endogenous Electrical Field in Vivo. Molecular Biology of the Cell, 25, 3835-3850. https://doi.org/10.1091/mbc.e13-12-0708

[35] Pietronero, L., Erzan, A. and Evertsz, C. (1988) Theory of Laplacian Fractals: Diffusion Limited Aggregation and Dielectric Breakdown Model. Physica A: Statistical Mechanics and Its Applications, 151, 207-245. https://doi.org/10.1016/0378-4371(88)90014-3

[36] Tokuyama, M. and Kawasaki, K. (1984) Fractal Dimensions for Diffusion-Limited Aggregation. Physics Letters A, 100, 337-340.

https://doi.org/10.1016/0375-9601(84)91083-1

[37] Whitaker, S. (1986) Flow in Porous Media I: A Theoretical Derivation of Darcy's Law. Transport in Porous Media, 1, 3-25. https://doi.org/10.1007/BF01036523

[38] Baish, J.W. and Jain, R.K. (2000) Fractals and Cancer. Cancer Research, 60, 3683-3688.

[39] Moon, B., Jagadish, H.V., Faloutsos, C. and Saltz, J.H. (2001) Analysis of the Clustering Properties of the Hilbert Space-Filling. IEEE Transactions on Knowledge and Data Engineering, 13, 124-141. https://doi.org/10.1109/69.908985

[40] Valova, I., Beaton, D., Buer, A. and Maclean, D. (2010) Fractal Initialization for High-Quality Mapping with Self-Organizing Maps. Neural Computing and Applications, 19, 953-966. https://doi.org/10.1007/s00521-010-0413-5

[41] Murray, J. (2003) Mathematical Biology II. Springer Verlag, New York, 548.

[42] Meakin, P. (1983) Diffusion-Controlled Cluster Formation in 2-6-Dimensional Space. Physical Review A, 27, 1495-1507. https://doi.org/10.1103/PhysRevA.27.1495

[43] Meakin, P., Majid, I., Havlin, S. and Stanley, H.E. (1984) Topological Properties of Diffusion Limited Aggregation and Cluster-Cluster Aggregation. Journal of Physics $A$, 17, L975-L981. https://doi.org/10.1088/0305-4470/17/18/008

[44] West, G.B., Brown, J.H. and Enquist, B.J. (1999) The Four Dimension of Life: Fractal Geometry and Allometric Scaling of Organisms. Science, 284, 1677-1679. https://doi.org/10.1126/science.284.5420.1677

[45] Warburg, O. (1956) On the Origin of Cancer Cells. Science, 123, 309-314. https://doi.org/10.1126/science.123.3191.309 